\title{
A new mouse-eared bat (Mammalia: Chiroptera: Vespertilionidae) from Vietnam
}

\author{
Alex V. Borisenko, Sergei V. Kruskop \& Natalia V. Ivanova
}

\begin{abstract}
A new mouse-eared bat (Mammalia: Chiroptera: Vespertilionidae) from the Myotis "siligorensis" species group is being described from the Hon Ba Mountain, ca. $30 \mathrm{~km}$ WSW of Nha Trang, Khanh Hoa Province, Vietnam $\left(12.1113^{\circ} \mathrm{N}, 108.953^{\circ} \mathrm{E}, 1250 \mathrm{~m}\right.$ ASL), based on a set of morphological and genetic characters. The new species is essentially similar to M. siligorensis alticraniatus, differing in slightly larger size, morphometrics, fine cranial and bacular traits. $12 \mathrm{~S}$ rDNA demonstrates ca. 2\% sequence divergence between the new species and its nearest neighbour, suggesting a history of genetic isolation. Provisional bat survey data from the Bi Doup-Hon Ba massif suggest that, although the new species co-occurs with $M$. siligorensis in the southern part of the Vietnam Central Highlands area, they are separated by an altitudinal gradient and habitat preferences, the former occupying mature forest at higher elevations and the latter confined to disturbed foothill areas.
\end{abstract}

KEY WORDS: Myotis, new species, Chiroptera, Vespertilionidae, Vietnam.

Alex V. Borisenko [aborisen@uoguelph.ca], Biodiversity Institute of Ontario, University of Guelph, Guelph, Ontario, Canada N1G 2W1; Sergei V. Kruskop [kruskop@zmmu.msu.ru], Zoological Museum, Moscow State University, Bolshaya Nikitskaya, 6, Moscow 125009, Russia; Natalia V. Ivanova [nivanova@uoguelph.ca], Biodiversity Institute of Ontario, University of Guelph, Guelph, Ontario, Canada N1G 2W1.

\section{Новый вид летучей мыши (Mammalia: Chiroptera: Vespertilionidae) из Вьетнама}

\begin{abstract}
А.В. Борисенко, С.В. Крускоп, Н.В. Иванова
РЕЗЮМЕ. Новый вид ночниц (Mammalia: Chiroptera: Vespertilinidae) из группы видов Myotis “siligorensis", описан из окрестностей горы Хон Ба, 30 км ЗЮЗ от г. Нячанг, пров. Кхань Хоа, Вьетнам $\left(12.1113^{\circ}\right.$ с.ш., $108.953^{\circ}$ в.д., 1250 м н.у.м.), на основе ряда морфологических и генетических признаков. Эта ночница в целом сходна с M. siligorensis alticraniatus, отличаясь несколько бьльшими средними размерами, морфометрически, а также особенностями строения черепа и бакулюма. Отличия от ближайшего родственного вида по последовательности митохондриальной $12 \mathrm{~S}$ рДНК составляют около $2 \%$, указывая на достаточно длительную генетическую изоляцию. Результаты предварительного обследования массива Би Дуп-Хон Ба указывают что, хотя описываемый вид и M. siligorensis симпатричны в южной части Центрального нагорья Вьетнама, они расходятся по высотной поясности и биотопическим предпочтениям: первый населяет спелый лес в высокогорной части, в то время как второй приурочен к нарушенным ландшафтам предгорий.
\end{abstract}

КЛЮЧЕВЫЕ СЛОВА: Myotis, новый вид, рукокрылые, гладконосые, Вьетнам.

\section{Introduction}

The taxonomy of the genus Myotis - one of the largest genera among living mammals (Simmons, 2005) - is highly complex and its understanding remains limited, especially with respect to small-sized tropical representatives with restricted or sporadic distribution and limited representation in collections. For example, the distinction between the "siligorensis" and "longipes" species groups can be difficult, despite the fact that their nominotypical species, M. siligorensis (Horsfield, 1855) and M. longipes (Dobson, 1873), were traditionally placed into two separate subgenera Selysius and Leuconoe, respectively (Tate, 1941; Ellerman \& Morrison-Scott, 1966; Koopman, 1994). The recent description of two new species within the "longipes" group (Topal, 1997, Kruskop \& Tsytsulina, 2000) suggests taxonomic diversity of these small bats remains partially undescribed.

In spring 2002 a collection of bats was taken by AVB during a baseline ecological survey in the southern part of the Vietnam Central Highlands, which contained several specimens of small mouse-eared bats, provisionally identified as " $M$. cf. alticraniatus" (Borisenko et al., 2006). An in-depth comparison between the specimens captured at different elevations within this area suggested the presence of two morphological types differing by altitudinal gradient. This was later corroborated by analyses of mitochondrial DNA, which revealed the presence of two distinct haplo- 
groups. Subsequent examination of collection materials on different tropical Asian Myotis stored in the Royal Ontario Museum and part of the type series of Myotis siligorensis alticraniatus Osgood, 1938 preserved in the Field Museum of Natural History suggested that the morphotype inhabiting the lowlands essentially resembles Myotis siligorensis from other parts of South-East Asia, while the highland form demonstrates morphological peculiarities, justifying its description as a distinct species.

\section{Materials and methods}

Study site. Bat collecting was conducted in April 2003 and concentrated around two sites within the proposed Hon Ba Mountain National Park, ca. 30 km WSW of Nha Trang, Khanh Hoa Province, Vietnam: near the mountain top $\left(12.12^{\circ} \mathrm{N}, 108.95^{\circ} \mathrm{E}\right.$; ca. 1200 $1500 \mathrm{~m} \mathrm{ASL}$ ) and in the foothills in several locations along Suoi Dau River and its right tributary $\left(12.12^{\circ} \mathrm{N}\right.$, $109.00^{\circ} \mathrm{E}$, ca. $200-250 \mathrm{~m}$ ASL). During the study period, which coincided with the end of the dry season, the water level in montane and lowland rivers was relatively low.

Collecting methods. Both mist nets (Jones et al., 1996) and a flap trap (mobile trap) (Borissenko, 1999; Borissenko \& Kruskop, 2003) were used to capture bats in flight, however, only the latter method proved to be successful. All captured bats were taken near or directly above streams within the first three hours following sunset.

Specimen processing. Captured bats were euthanized in the vapours of chloroform, following recommended protocols (American Society of Mammalogists Animal Care and Use Committee, 1998). Four external measurements (head and body, tail, ear and forearm length) were taken post-mortem with vernier calipers. Fixation in $75 \%$ ethanol followed within 10 to 15 minutes after euthanasia. Subsequent processing of collection material resumed within several months following fixation. External measurements were taken with vernier or electronic calipers to the nearest $0.1 \mathrm{~mm}$. Skulls of seven specimens were extracted and cleared in a dermestarium. Cleaned skulls were measured under a dissecting microscope with electronic calipers to the nearest $0.01 \mathrm{~mm}$. Tissue samples for DNA extraction were not collected on the day of capture and had to be taken at a later date from muscle of alcohol-preserved specimens.

Comparative material. Forty seven specimens of morphologically similar species were used for qualitative and quantitative morphological comparison (adult individuals of both sexes; dry or alcohol preserved skins with extracted skulls). Acronyms of repositories of the processed collections are as follows: FMNH Field Museum of Natural History, Chicago; ROM Royal Ontario Museum, Toronto; ZMMU - Zoological Museum of Moscow State University, Moscow.

Myotis csorbai Topal, 1997: Central Nepal (including five paratypes of the species): ZMMU S-164475 f,
ZMMU S-164476 f, ZMMU S-164478 f, ZMMU S164481 f, ZMMU S-164483 f, ZMMU S-164484 f, ZMMU S-164485 m, ZMMU S-164487 m, ZMMU S$164490 \mathrm{~m}$.

Myotis cf. csorbai: China, Sichuan: FMNH 39571 m, FMNH 39572 m.

Myotis annamiticus Kruskop \& Tsytsulina, 2000: Central Vietnam (from the type series of the species): ZMMU S-167123 m, ZMMU S-167126 m, ZMMU S167127 f, ZMMU S-167128 f, ZMMU S-167132 f, ZMMU S-167134 f, ZMMU S-167135 f.

Myotis siligorensis alticraniatus: Hon Ba, Vietnam Central Highlands: ZMMU S-175152 f, ZMMU S175157 m, ZMMU S-175159 f; Quang Binh, Central Vietnam: ZMMU S-167188 m; Northern Vietnam (Tuen Quang, Sa Pa and Muon Muon; including three females from the type series): ROM MAM $107649 \mathrm{f}$, ROM MAM 107652 f, ROM MAM 107657 f, ROM MAM 107677 f, ROM MAM 112447 m, ROM MAM 112456 m, ROM MAM 112458 m, FMNH 32173 f, FMNH 32175 f, FMNH $32178 \mathrm{f}$.

Myotis laniger (Peters, 1871): Southern China: ROM MAM 114940 m, ROM MAM 114955 f, ROM MAM 114968 m, ROM MAM 15058 f; Northern Vietnam: ROM MAM 106167 m, ROM MAM 107665 f, ROM MAM 107712 m, ROM MAM 112430 m, ROM MAM 112492 m, ROM MAM 111269 m, ROM MAM 111284 m; specimens from Fujian, South-East China, originally labeled as “ $M$. siligorensis sowerbyi”: FMNH 33858 m, FMNH 33859 m, FMNH 44109 f.

In addition to this, qualitative comparisons were made with ROM and ZMMU collection materials representing Myotis species of similar size classes occurring in the region (M. muricola, M. horsfieldii, $M$. hasseltii, $M$. cf. ater) and select species occurring extralimtally (M. longipes, M. petax, M. macrodactylus, M. mystacinus, M. brandtii, M. ikonnikovi), in order to confirm their dissimilarity.

Fourteen ZMMU specimens were used in molecular analyses of 12S mtDNA: $M$. montivagus S-164998, $M$. csorbai S-164485, M. rossetii S-175291, M. siligorensis alticraniatus S-175152, M. new species S-175154, M. annamiticus S-167125, M. hasseltii S-174723, M. horsfieldii S-165039, M. muricola S-175134, Murina huttoni S-175150, Kerivoula hardwickii S-175110, K. titania S-175149, Eudiscopus denticulus S-172558. The following $12 \mathrm{~S}$ sequences were taken from NCBI GenBank: M. siligorensis AY495508.1, M. capaccinii AY495494.1, M. adversus AY495291.1, M. muricola AY495504.1, and M. rossettii AY495505.1 (specimen deposited in GenBank as M. ridleyi).

Morphometric analyses. The following external measurements were taken: forearm length, tibia length, foot length (including claws, measured to the distal extremity of claw), length of the first digit (including claw), length of the metacarpal of the second digit, and lengths of the metacarpals and phalanges of the third, through and fifth digits. All wing measurements were taken on the right wing, unless unsuitable (e.g., dam- 
aged) or inaccessible for measuring. The following cranial measurements, (abbreviations given in parentheses), were taken: condylobasal length (CBL), condylocanine length (CCL), rostral length from the anterorbital foramen to the alveolus of the inner incisor (RL), occiput height $(\mathrm{OccH})$, tympanic width of skull at the level of the auditory bullae (TW), width of braincase (BCW); least interorbital width (IOW), rostral width at the level of the infraorbital foramina (WR), length of interval between the cingula of the upper canine and large premolar («pseudodiastem», Pseud), C-M3 length (CM3), molariform row length (P4M3), length of the upper canine cingulum base $(\mathrm{C})$, length of interval between tips of upper canine and large premolar (CP4), crown measured width between outer margins of upper canines (CC), crown measured width between outer margins of M3 (M3M3), width of M2 (M2), lower jaw length from alveolus of $\mathrm{i} 1$ to the glenoid process (Mnd gl), lower jaw length from alveoli of i1 to the angular process (Mnd ang), crown length of maxillary tooth row $(\mathrm{cm} 3)$, crown length of the maxillary molariform row (p4m3), length of interval between the tips of the lower canine and the large premolar (cp4), lower jaw height to the tip of the coronoid process (HMnd).

To assess the pattern of variation of quantitative characters, Discriminant Function (DF) and Principal Component (PC) analyses were performed for cranial measurements, using the Discriminant Function and Factor Analysis modules of STATISTICA for Windows (StatSoft, Inc., 1999). For PC analysis, measurements were standardized (row score-mean/standard deviation) to decrease the influence of the overall size. DF analysis was used to calculate squared Mahalanobis distances between groups.

Molecular analyses. $12 \mathrm{~S} \mathrm{mt}$ rDNA was analyzed at the Engelhardt Institute of Molecular Biology (Moscow, Russia). Total DNA from ethanol-preserved tissue was extracted using commercially available kits: NucleoSorb (Biokom, Russia), based on guanidine thiocyanate-diatomite method, or ExtraGene (Biokom, Russia) based on lysis in the presence of proteolytic enzymes and ion and anion-exchange resins. Two primer pairs were used to amplify two overlapping fragments of 12S rDNA (Kuznetsov et al., 2001): 12c 5'-aaagcaaarcactgaaaatg-3' (Springer et al., 1995) with mt12Sr RNA860H 5'-ggtatctaatcccagtttg-3', and mt12SrRNA 670L 5'-cagccaccgcgg[t/c]catac-3' with 12 g 5' - ttcatctt ttccttgcggtac-3'(Springer, et al., 1995). PCR reactions were performed as described in Kuznetsov et al. (2001). PCR products were sequenced using an ABI Prism BigDye Terminator v 3.1. Cycle Sequencing kit and analyzed on an ABI 3100 Genetic Analyzer (Applied Biosytems). Subsequent proofreading and assembling of sequences were done in Lasergene software (DNASTAR). Subsequent proofreading and assembling of sequences were done in Lasergene software (DNASTAR). Sequences were aligned in BioEdit 7.0.5.3 (Hall, 1999) software using the ClustalW algorithm with the following settings: Gap opening penalty 10, Gap extension penalty 5 , and manually adjusted.
The 658 base pair 5' segment of the cytochrome oxidase subunit I (COI) gene (standard DNA barcode region) was analyzed at the Canadian Centre for DNA Barcoding, Biodiversity Institute of Ontario, (Guelph, Canada). Whole genomic DNA from ethanol-preserved tissue was recovered using an automated DNA extraction method (Ivanova et al., 2006). PCR amplification for the standard DNA barcode region of $657 \mathrm{bp}$ using M13-tailed primer cocktails C_VF1LFt1 and C_VR1LRt1 (Ivanova et al., 2007) was performed as described in Clare et al. (2007), Borisenko et al. (2008). For degraded samples we obtained 421 bp the using M13-tailed RonM primer (Pfunder et al., 2004) in combination with the reverse mammal cocktail C_VR1LRt1 as described in Borisenko et al. (2008). PCR products were sequenced using an ABI Prism BigDye Terminator v 3.1. Cycle Sequencing kit and analyzed on ABI 3730XL Genetic Analyzer (Hajibabaei et al., 2005). Bidirectional reads were assembled and manually edited in SEQSCAPE 2.1.1 software (Applied Biosystems).

$12 \mathrm{~S}$ Sequences are deposited in NCBI GenBank (GenBank accession numbers: FJ755892-FJ755904). DNA barcode data (COI sequences, chromatogram trace files, and collateral specimen information) are stored in the Barcode of Life Data System (Ratnasingham, Hebert, 2007) at http://www.barcodinglife.org in the Completed Projects section in the project titled "Myotis phanluongi sp. nov. [ABMPH]"; they are also deposited in NCBI GenBank (accession numbers FJ408723 FJ408729).

Analysis of molecular $12 \mathrm{~S}$ data was performed using MEGA4 molecular genetic analysis software (Tamura et al., 2007). A distance-based tree was built with the Neighbour-Joining algorithm using the Maximum Composite Likelihood method and pairwise deletion of missing data. A character-based tree was inferred with the Maximum Parsimony method using the Close-Neighbour-Interchange algorithm with search level 7 in which the initial trees were obtained with the random addition of sequences (10 replicates). Alignment gaps were treated as missing data. The tree was rooted with the composite outgroup containing Murina and Kerivoula specimens. There were a total of 991 positions in the final dataset, of which 201 were parsimony informative. In both methods branch support was assessed by bootstrapping with 1000 replicates.

\section{Results and discussion}

\section{Myotis phanluongi Borisenko, Kruskop et Ivanova, species nova}

Holotype. ZMMU S-175155 (alcoholic, skull and baculum extracted), adult male; coll. A.V. Borisenko, 20 April 2003.

Type locality. Hon Ba Mountain, ca. $30 \mathrm{~km}$ WSW of Nha Trang, Khanh Hoa Province, Vietnam $\left(12.1113^{\circ} \mathrm{N}\right.$, $108.953^{\circ} \mathrm{E}, 1250 \mathrm{~m} \mathrm{ASL}$ ). 


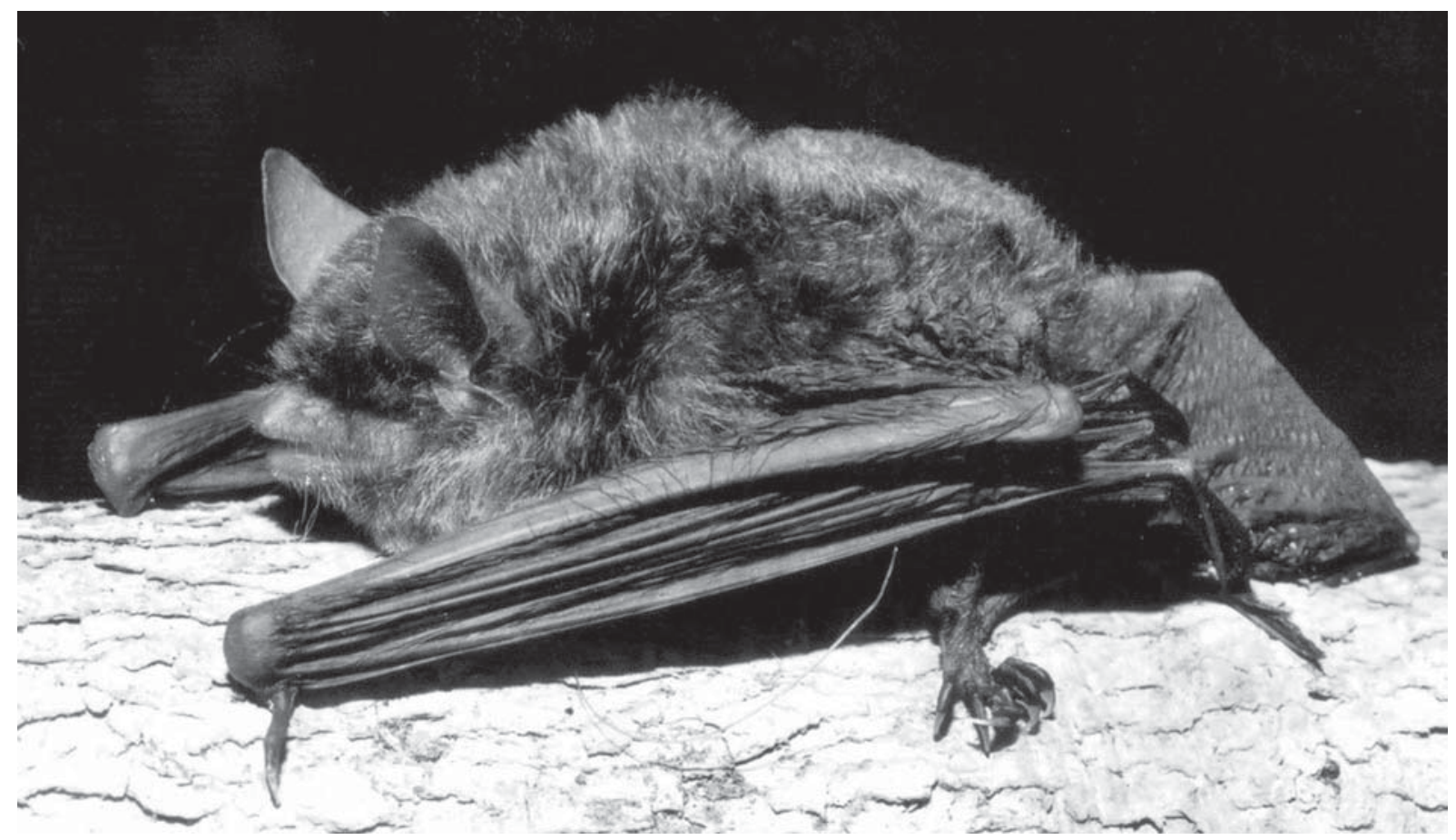

Figure 1. Live photograph of Myotis phanluongi sp. nov., male (holotype); 20 April 2003, Hon Ba.

Paratypes. Three adult females, ZMMU S-175153, S-175154 (alcoholic, skulls extracted; in 175154 tissue sample taken), same date, locality and collector; S175156 (alcoholic, skull extracted, damaged); same general locality $\left(12.1144^{\circ} \mathrm{N}, 108.955^{\circ} \mathrm{E}, 1275 \mathrm{~m}\right)$ and collector, 25 April 2003.

Other specimens. One adult female, ZMMU S182120, collected in the Da Nim River valley, $5 \mathrm{~km} \mathrm{NE}$ from Long Lanh, Lam Dong Prov., Vietnam $\left(12.179^{\circ} \mathrm{N}\right.$, $108.679^{\circ} \mathrm{E}, 1400 \mathrm{~m}$ ASL), 13 April 2008.

Diagnosis. Essentially similar in external, cranial and dental qualitative characters to $M$. siligorensis alticraniatus (Figs 1 and 2), but possessing slightly larger overall size and cranial dimensions, which are more similar to those of M. annamiticus (Tab. 1). Externally differs from $M$. s. alticraniatus in slightly paler muzzle and limbs. Rostral part of skull relatively longer than in $M$. siligorensis and $M$. annamiticus, and concaved upwards (Fig. 2A). Lower molar cusp pattern seminyctalodont (sensu Ziegler, 2003), similar to M. siligorensis and contrary to most other Myotis species. Baculum distinctive from both $M$. siligorensis and $M$. annamiticus in having an almost peg-like appearance, lacking urethral groove or any concavity on ventral side (Fig. 3A).

DNA barcode of the holotype. NCBI GenBank accession FJ408724, BOLD (http://www.barcofinglife. org) process ID BM625-04.

Measurements of the holotype (in mm). External measurements, (post-mortem): head \& body 39, tail 35, forearm 33.5, tibia13.5, hind foot 7.4, ear pinna 12.3, tragus 6.7, wing span 223. Skull measurements: CBL 12.01, CCL 11.20, RL 4.14, OccH 5.02, TW 6.39,
BCW 5.97; IOW 2.84, WR 4.78, Pseud 0.76, C-M3 4.92, P4-M3 3.49, C 0.66, CP4 1.42, CC 3.15, M3-M3 4.92, M2 1.16, Mnd g1 9.07, Mnd ang 9.30, cm3 5.14, p4m33.69, cp4 1.33, HMnd 2.45.

Etymology. The new species is named in honour of Dr. Phan Luong (1948-2008), head of the Laboratory of Ecology of the Vietnamese-Russian Tropical Centre, to acknowledge his key role in organizing numerous ecological survey expeditions across Vietnam, including this field trip to Hon $\mathrm{Ba}$, and to commemorate his important contribution towards the study of Vietnamese tropical forest ecosystems.

Description. A small-sized Myotis: forearm length 33.5-36.0 mm (average 34.9; $\mathrm{n}=4$ ), condylobasal length of skull 12.01-12.80 mm (average 12.53; $\mathrm{n}=3$ ). Margin of plagiopatagium attached to the distal part of the metatarsus, just proximal to the basal joint of the outer toe. Skull with a low and slender, fairly elongated rostrum and nearly globular elevated braincase (Fig. 2A). Cranial profile gradually rising in interorbital region; rostral part of skull slightly curved upwards, frontal part of braincase distinctly elevated. Upper canine short, not exceeding height of $\mathrm{P} 4$, round in cross-section; canine cingulum completely encircling the crown (Fig. 4A). Both small upper premolars (P2 and P3) are loosely positioned within tooth row (sometimes not in contact) and are similar to each other in shape (P3 smaller than $1 / 2$ height of P2). Pseudodiastem (gap between canine and molariform premolar) slightly exceeding cingular length of canine. $\mathrm{P} 4$ with distinctive anterolingual accessory cusp. M2 and M3 with wellpronounced paraconules on the preprotocristae. Lower 
Table 1. Cranial measurements of Myotis phanluongi sp. nov. and morphologically similar species (mean[ \pm standard deviation if $n>3$ ] min-max). See text for measurement abbreviations.

\begin{tabular}{|c|c|c|c|c|c|c|c|}
\hline & \begin{tabular}{|l|}
. \\
phanluongi \\
sp.n, $\mathrm{n}=3$
\end{tabular} & $\begin{array}{l}\text { M. } \text { csorbai } \\
\mathrm{n}=9\end{array}$ & $\begin{array}{l}\text { M. cf. csorbai } \\
\mathrm{n}=2\end{array}$ & $\begin{array}{l}\text { M. annamiticus } \\
\mathrm{n}=7\end{array}$ & $\begin{array}{l}\begin{array}{l}\text { M. siligorensis } \\
\mathrm{n}=13\end{array} \\
\text { 作 }\end{array}$ & $\begin{array}{l}\text { M. laniger } \\
\mathrm{n}=11\end{array}$ & $\begin{array}{l}\text { "M. sowerbyi" } \\
\mathrm{n}=3\end{array}$ \\
\hline CBL & \begin{tabular}{|l|}
12.53 \\
$12.01-12.8$ \\
\end{tabular} & $\begin{array}{l}12.29 \pm 0.35 \\
11.7-12.78 \\
\end{array}$ & $11.58-11.59$ & \begin{tabular}{|l|}
$12.18 \pm 0.11$ \\
$12.00-12.34$ \\
\end{tabular} & \begin{tabular}{|l|}
$11.22 \pm 0.22$ \\
$10.89-11.63$ \\
\end{tabular} & \begin{tabular}{|l|}
$13.08 \pm 0.46$ \\
$12.06-13.74$ \\
\end{tabular} & \begin{tabular}{|l|}
11.99 \\
$11.5-12.58$ \\
\end{tabular} \\
\hline CCL & \begin{tabular}{|l|}
11.67 \\
$11.2-11.94$ \\
\end{tabular} & \begin{tabular}{|l|}
$11.57 \pm 0.33$ \\
$11.09-12.03$ \\
\end{tabular} & $10.92-10.93$ & \begin{tabular}{|l|}
$11.48 \pm 0.09$ \\
$11.39-11.64$ \\
\end{tabular} & \begin{tabular}{|l|}
$10.52 \pm 0.2$ \\
$10.24-10.84$ \\
\end{tabular} & \begin{tabular}{|l|}
$12.38 \pm 0.44$ \\
$11.43-12.99$ \\
\end{tabular} & \begin{tabular}{|l|}
11.32 \\
$10.82-11.81$ \\
\end{tabular} \\
\hline RostrL & $\begin{array}{l}4.40 \\
4.14-4.53\end{array}$ & $\begin{array}{l}3.85 \pm 0.2 \\
3.52-4.07\end{array}$ & $3.76-3.91$ & $\begin{array}{l}3.93 \pm 0.17 \\
3.76-4.27\end{array}$ & $\begin{array}{l}3.8 \pm 0.11 \\
3.62-3.97\end{array}$ & $\begin{array}{l}4.39 \pm 0.27 \\
3.73-4.70\end{array}$ & \begin{tabular}{|l|}
3.75 \\
$3.61-3.89$
\end{tabular} \\
\hline $\mathrm{OccH}$ & \begin{tabular}{|l|}
5.02 \\
$5.02-5.03$ \\
\end{tabular} & \begin{tabular}{|l|}
$5.3 \pm 0.08$ \\
$5.16-5.41$ \\
\end{tabular} & $5.12-5.21$ & $\begin{array}{l}5.31 \pm 0.06 \\
5.23-5.40 \\
\end{array}$ & $\begin{array}{l}4.86 \pm 0.09 \\
4.71-5.00 \\
\end{array}$ & $\begin{array}{l}5.69 \pm 0.22 \\
5.30-6.01 \\
\end{array}$ & \begin{tabular}{|l|}
5.45 \\
$5.17-5.67$ \\
\end{tabular} \\
\hline TympW & \begin{tabular}{|l|}
6.54 \\
$6.39-6.62$ \\
\end{tabular} & \begin{tabular}{|l|}
$6.73 \pm 0.23$ \\
$6.41-6.99$ \\
\end{tabular} & 6.69-6.72 & \begin{tabular}{|l|}
$6.68 \pm 0.13$ \\
$6.51-6.92$ \\
\end{tabular} & \begin{tabular}{|l|}
$6.34 \pm 0.12$ \\
$6.11-6.52$ \\
\end{tabular} & $\begin{array}{l}7.17 \pm 0.22 \\
6.70-7.51 \\
\end{array}$ & \begin{tabular}{|l|}
7.00 \\
$6.86-7.18$ \\
\end{tabular} \\
\hline BrainW & \begin{tabular}{|l|}
6.14 \\
$5.97-6.33$ \\
\end{tabular} & \begin{tabular}{|l|}
$6.51 \pm 0.16$ \\
$6.22-6.74$ \\
\end{tabular} & $6.38-6.48$ & \begin{tabular}{|l}
$6.56 \pm 0.13$ \\
$6.40-6.79$ \\
\end{tabular} & \begin{tabular}{|l|}
$5.98 \pm 0.13$ \\
$5.69-6.15$ \\
\end{tabular} & $\begin{array}{l}7.06 \pm 0.24 \\
6.58-7.36 \\
\end{array}$ & \begin{tabular}{|l|}
6.64 \\
$6.40-6.87$ \\
\end{tabular} \\
\hline IOrbW & \begin{tabular}{|l|}
2.89 \\
$2.84-2.94$ \\
\end{tabular} & \begin{tabular}{|l|}
$3.24 \pm 0.11$ \\
$3.11-3.46$ \\
\end{tabular} & $3.16-3.19$ & $\begin{array}{l}3.20 \pm 0.05 \\
3.13-3.25 \\
\end{array}$ & & $\begin{array}{l}3.4 \pm 0.11 \\
3.23-3.54 \\
\end{array}$ & \begin{tabular}{|l|}
3.27 \\
$3.12-3.49$ \\
\end{tabular} \\
\hline RostrW & \begin{tabular}{|l|}
4.95 \\
$4.78-5.08$ \\
\end{tabular} & \begin{tabular}{|l|}
$4.90 \pm 0.11$ \\
$4.74-5.12$ \\
\end{tabular} & $4.31-4.56$ & \begin{tabular}{|l|}
$4.69 \pm 0.06$ \\
$4.56-4.74$ \\
\end{tabular} & & \begin{tabular}{|l|}
$5.07 \pm 0.23$ \\
$4.70-5.49$ \\
\end{tabular} & \begin{tabular}{|l|}
4.53 \\
$4.27-4.78$ \\
\end{tabular} \\
\hline Pseud & \begin{tabular}{|l|}
0.77 \\
$0.76-0.77$ \\
\end{tabular} & $\begin{array}{l}0.54 \pm 0.08 \\
0.43-0.63 \\
\end{array}$ & $0.57-0.67$ & $\begin{array}{l}0.83 \pm 0.05 \\
0.78-0.92 \\
\end{array}$ & $\begin{array}{l}0.72 \pm 0.07 \\
0.61-0.84 \\
\end{array}$ & $\begin{array}{l}0.92 \pm 0.07 \\
0.83-1.09 \\
\end{array}$ & \begin{tabular}{|l|}
0.76 \\
$0.69-0.86$ \\
\end{tabular} \\
\hline $\mathrm{C}-\mathrm{M} 3$ & \begin{tabular}{|l|}
5.12 \\
$4.92-5.28$
\end{tabular} & \begin{tabular}{|l|}
$4.95 \pm 0.15$ \\
$4.64-5.14$
\end{tabular} & 4.56 & $\begin{array}{l}4.89 \pm 0.08 \\
4.80-5.06\end{array}$ & & \begin{tabular}{|l|}
$5.44 \pm 0.28$ \\
$4.69-5.71$
\end{tabular} & \begin{tabular}{|l|}
4.72 \\
$4.51-4.91$
\end{tabular} \\
\hline P4-M3 & \begin{tabular}{|l|}
3.61 \\
$3.49-3.72$ \\
\end{tabular} & \begin{tabular}{|l|}
$3.66 \pm 0.12$ \\
$3.45-3.80$ \\
\end{tabular} & $3.32-3.38$ & \begin{tabular}{|l|}
$3.36 \pm 0.06$ \\
$3.28-3.49$ \\
\end{tabular} & \begin{tabular}{|l|}
$3.10 \pm 0.08$ \\
$2.97-3.23$ \\
\end{tabular} & \begin{tabular}{|l|}
$3.76 \pm 0.19$ \\
$3.29-3.93$ \\
\end{tabular} & \begin{tabular}{|l|}
3.34 \\
$3.19-3.41$ \\
\end{tabular} \\
\hline C-crown & \begin{tabular}{|l|}
0.70 \\
$0.66-0.73$ \\
\end{tabular} & \begin{tabular}{|l|}
$0.75 \pm 0.04$ \\
$0.68-0.81$ \\
\end{tabular} & $0.63-0.64$ & \begin{tabular}{|l|}
$0.68 \pm 0.01$ \\
$0.66-0.7$ \\
\end{tabular} & \begin{tabular}{|l|}
$0.59 \pm 0.06$ \\
$0.51-0.70$ \\
\end{tabular} & $\begin{array}{l}0.80 \pm 0.07 \\
0.61-0.85 \\
\end{array}$ & \begin{tabular}{|l|}
0.65 \\
$0.61-0.69$ \\
\end{tabular} \\
\hline $\mathrm{C}-\mathrm{P} 4$ & \begin{tabular}{|l|}
1.52 \\
$1.42-1.57$ \\
\end{tabular} & \begin{tabular}{|l|}
$1.34 \pm 0.07$ \\
$1.22-1.45$ \\
\end{tabular} & $1.06-1.16$ & \begin{tabular}{|l|}
$1.55 \pm 0.07$ \\
$1.44-1.63$ \\
\end{tabular} & \begin{tabular}{|l|}
$1.24 \pm 0.1$ \\
$1.12-1.40$ \\
\end{tabular} & \begin{tabular}{|l|}
$1.65 \pm 0.11$ \\
$1.37-1.81$ \\
\end{tabular} & \begin{tabular}{|l|}
1.24 \\
$1.19-1.31$ \\
\end{tabular} \\
\hline $\mathrm{C}-\mathrm{C}$ & \begin{tabular}{|l|}
3.24 \\
$3.15-3.39$ \\
\end{tabular} & \begin{tabular}{|l|}
$3.41 \pm 0.13$ \\
$3.27-3.62$ \\
\end{tabular} & $3.20-3.25$ & \begin{tabular}{|l|l|}
$3.31 \pm 0.1$ \\
$3.19-3.45$ \\
\end{tabular} & \begin{tabular}{|l|}
$2.99 \pm 0.08$ \\
$2.84-3.15$ \\
\end{tabular} & \begin{tabular}{|l|}
$3.54 \pm 0.24$ \\
$3.05-3.84$ \\
\end{tabular} & \begin{tabular}{|l|}
3.22 \\
$3.03-3.40$ \\
\end{tabular} \\
\hline M3-M3 & \begin{tabular}{|l|}
5.17 \\
$4.92-5.37$ \\
\end{tabular} & \begin{tabular}{|l|}
$5.16 \pm 0.17$ \\
$4.90-5.39$ \\
\end{tabular} & $5.14-5.23$ & \begin{tabular}{|l|}
$4.92 \pm 0.07$ \\
$4.79-5.00$ \\
\end{tabular} & \begin{tabular}{|l|}
$4.78 \pm 0.52$ \\
$4.38-6.47$ \\
\end{tabular} & $\begin{array}{l}5.40 \pm 0.22 \\
5.06-5.76 \\
\end{array}$ & \begin{tabular}{|l|}
4.93 \\
$4.73-5.05$ \\
\end{tabular} \\
\hline M2 & \begin{tabular}{|l|}
1.33 \\
$1.16-1.48$ \\
\end{tabular} & \begin{tabular}{|l|}
$1.34 \pm 0.05$ \\
$1.28-1.40$ \\
\end{tabular} & $1.16-1.24$ & \begin{tabular}{|l|}
$1.16 \pm 0.06$ \\
$1.07-1.26$ \\
\end{tabular} & \begin{tabular}{|l|}
$1.09 \pm 0.05$ \\
$0.98-1.19$ \\
\end{tabular} & \begin{tabular}{|l|}
$1.28 \pm 0.07$ \\
$1.18-1.40$ \\
\end{tabular} & \begin{tabular}{|l|}
1.15 \\
$1.03-1.22$ \\
\end{tabular} \\
\hline Mndgl & \begin{tabular}{|l|}
9.55 \\
$9.07-9.86$ \\
\end{tabular} & \begin{tabular}{|l|}
$9.48 \pm 0.29$ \\
$8.95-9.87$ \\
\end{tabular} & $8.83-8.95$ & \begin{tabular}{|l|}
$9.13 \pm 0.1$ \\
$8.95-9.28$ \\
\end{tabular} & \begin{tabular}{|l|}
$8.45 \pm 0.18$ \\
$8.17-8.81$ \\
\end{tabular} & \begin{tabular}{|l|}
$10.15 \pm 0.46$ \\
$9.21-10.69$ \\
\end{tabular} & \begin{tabular}{|l|}
9.12 \\
$8.72-9.59$ \\
\end{tabular} \\
\hline Mndang & \begin{tabular}{|l|}
9.71 \\
$9.30-9.93$ \\
\end{tabular} & \begin{tabular}{|l|}
$9.74 \pm 0.23$ \\
$9.33-10.05$ \\
\end{tabular} & $9.12-9.23$ & \begin{tabular}{|l|}
$9.31 \pm 0.1$ \\
$9.19-9.44$ \\
\end{tabular} & \begin{tabular}{|l|}
$8.69 \pm 0.22$ \\
$8.33-9.17$ \\
\end{tabular} & \begin{tabular}{|l|}
$10.46 \pm 0.5$ \\
$9.33-11.05$ \\
\end{tabular} & \begin{tabular}{|l|}
9.33 \\
$8.97-9.68$ \\
\end{tabular} \\
\hline $\mathrm{c}-\mathrm{m} 3$ & \begin{tabular}{|l|}
5.38 \\
$5.14-5.58$ \\
\end{tabular} & \begin{tabular}{|l|}
$5.24 \pm 0.15$ \\
$4.90-5.39$ \\
\end{tabular} & $4.59-4.71$ & $\begin{array}{l}5.12 \pm 0.09 \\
5.05-5.31 \\
\end{array}$ & \begin{tabular}{|l|}
$4.57 \pm 0.14$ \\
$4.43-4.91$ \\
\end{tabular} & $\begin{array}{l}5.70 \pm 0.4 \\
4.59-6.02 \\
\end{array}$ & \begin{tabular}{|l|}
4.89 \\
$4.67-5.11$ \\
\end{tabular} \\
\hline $\mathrm{p} 4-\mathrm{m} 3$ & \begin{tabular}{|l|}
3.83 \\
$3.69-3.95$ \\
\end{tabular} & \begin{tabular}{|l|}
$3.85 \pm 0.1$ \\
$3.67-3.96$ \\
\end{tabular} & $3.44-3.84$ & \begin{tabular}{|l|}
$3.51 \pm 0.05$ \\
$3.44-3.59$ \\
\end{tabular} & $\begin{array}{l}3.25 \pm 0.11 \\
3.10-3.51 \\
\end{array}$ & $\begin{array}{l}3.89 \pm 0.26 \\
3.37-4.14 \\
\end{array}$ & \begin{tabular}{|l|}
3.52 \\
$3.38-3.60$ \\
\end{tabular} \\
\hline $\mathrm{c}-\mathrm{p} 4$ & \begin{tabular}{|l|}
1.41 \\
$1.33-1.54$ \\
\end{tabular} & \begin{tabular}{|l|}
$1.23 \pm 0.06$ \\
$1.14-1.34$ \\
\end{tabular} & $1.02-1.08$ & \begin{tabular}{|l|}
$1.50 \pm 0.05$ \\
$1.43-1.57$ \\
\end{tabular} & \begin{tabular}{|l|}
$1.19 \pm 0.21$ \\
$0.90-1.74$ \\
\end{tabular} & \begin{tabular}{|l|}
$1.64 \pm 0.13$ \\
$1.36-1.88$ \\
\end{tabular} & \begin{tabular}{|l|}
1.16 \\
$1.03-1.35$ \\
\end{tabular} \\
\hline Coron & \begin{tabular}{|l|}
2.57 \\
$2.45-2.67$
\end{tabular} & $\begin{array}{l}2.53 \pm 0.11 \\
2.38-2.69\end{array}$ & $2.49-2.57$ & $\begin{array}{l}2.19 \pm 0.12 \\
1.93-2.27 \\
\end{array}$ & $\begin{array}{l}2.23 \pm 0.05 \\
2.13-2.30\end{array}$ & \begin{tabular}{|l|}
$2.75 \pm 0.12$ \\
$2.58-2.93$
\end{tabular} & $\begin{array}{l}2.50 \\
2.32-2.72\end{array}$ \\
\hline
\end{tabular}

jaw slender; coronoid process low, not rising above glenoid process. Lower canine very short, subequal to p4. Lower molar pattern seminyctalodont; postcristid connected chiefly to hypoconulid. Baculum very small $(0.40 \mathrm{~mm}$ in length and $0.18 \mathrm{~mm}$ in maximal width in the holotype) straight and peg-like, not especially widened in its basal half; its lower surface almost circular in cross-section, with no urethral groove or any concavity and no winglets in the basal part (Fig. 3A).
Comparison with similar species. Among the Southeast Asian bat taxa studied as comparative material, $M$. phanluong $i$ sp. nov. exhibits greatest morphological and genetic similarities with $M$. s. alticraniatus, with which it was initially confused (Borisenko et al., 2006). It also bears some resemblance with $M$. annamiticus and M. laniger (sensu Topal, 1997). Aside from the differences mentioned in the diagnosis, it is distinguished morphometrically and demonstrates genetic 

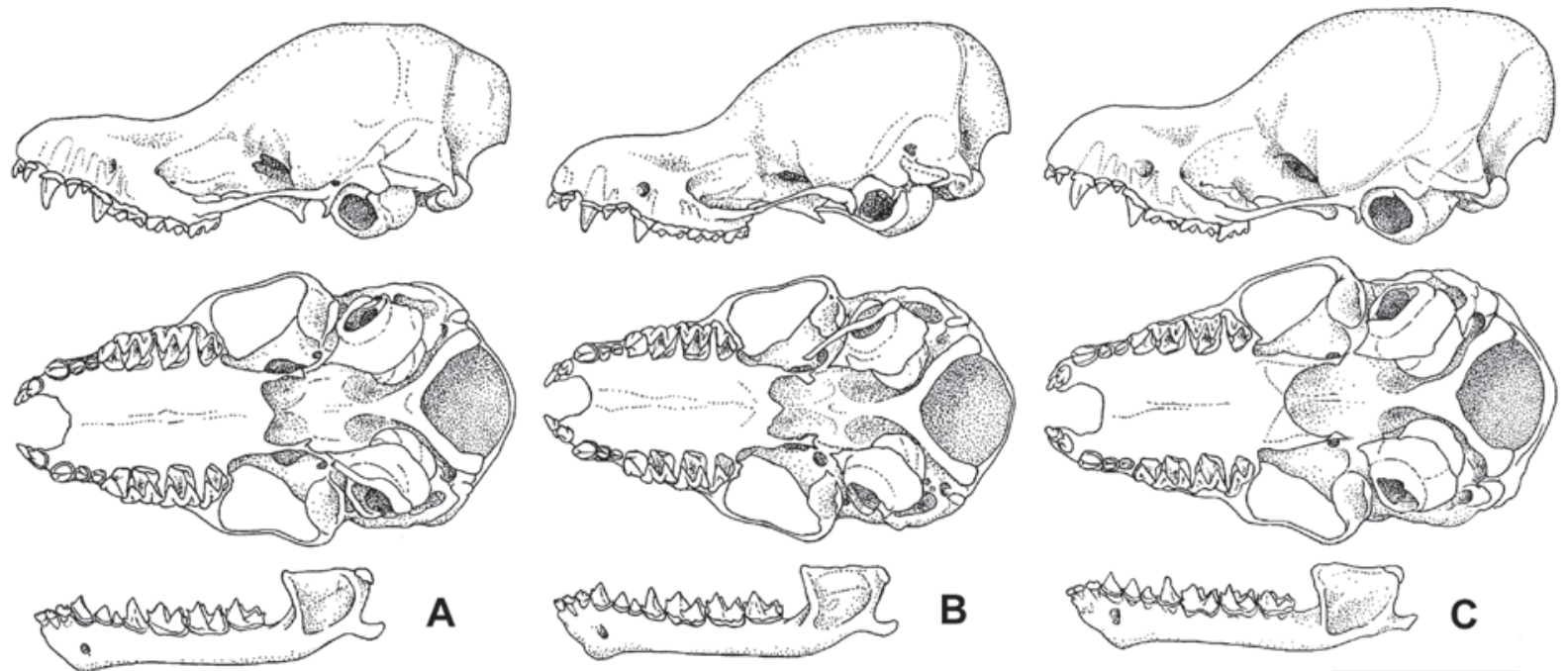

A
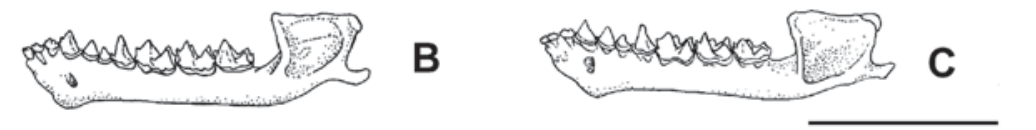

Figure 2. Camera lucida tracings of skulls of small Vietnamese Myotis (lateral view, ventral view, lower jaw, lateral view): A - M. phanluongi sp. nov., holotype ZMMU S-175155, male, Hon Ba; B - M. siligorensis alticraniatus, ZMMU S175157, Hon Ba; C - M. annamiticus, paratype ZMMU S-167135. Scale bar is $5 \mathrm{~mm}$.
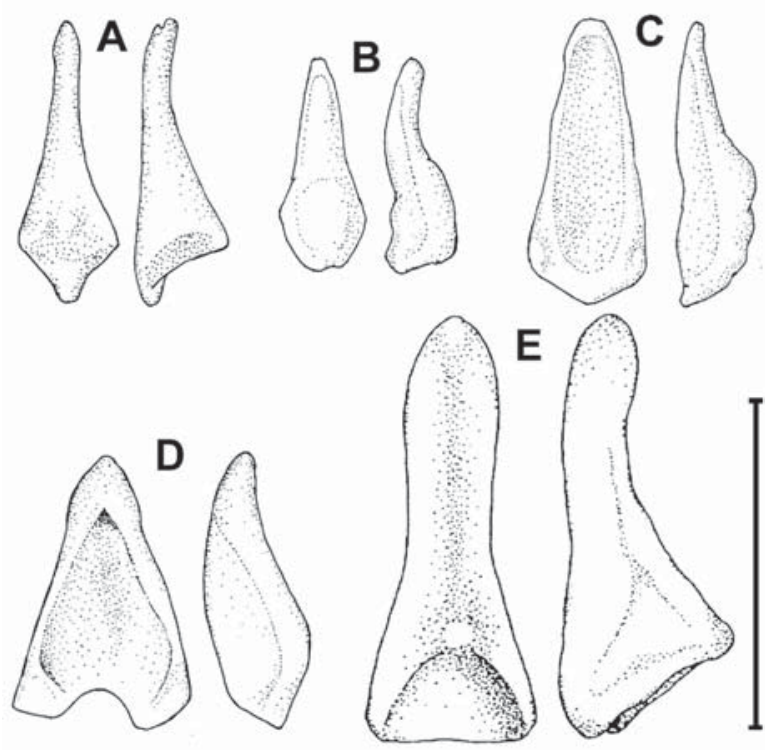

Figure 3. Camera lucida tracings of the bacula of selected small Myotis from Southeast Asia: A — Myotis phanluongi sp. nov., S-175155 (dorsal and left lateral view); B - Myotis siligorensis, S-175157 (dorsal and left lateral view); C Myotis siligorensis, S-167188 (ventral and left lateral view); D - Myotis csorbai, S-164485 (ventral and left lateral view); E - Myotis annamiticus (dorsal and left lateral view, after Kruskop \& Tsytsulina, 2001). Scale bar is $0.5 \mathrm{~mm}$.

divergence from the above taxa, discussed in more detail below.

The question remains open regarding the fine distinction of $M$. phanluongi sp. nov. from $M$. s. siligorensis (Horsfield, 1855) on the one hand and M. s. thaianus Shamel, 1942 on the other. Original collection material for these named forms has not been studied. The nominotypical form was described from "Nepal, Siligori" (Simmons, 2005), or, more appropriately, "Siliguri, West Bengal, India" (Bates \& Harrison, 1997). This is much more remote from the type locality of $M$. phanluongi sp. nov. than that of M. s. alticraniatus. Available images of the type specimen preserved at the British Museum (kindly provided by Charles Francis) suggest subtle differences in skull shape from both M. s. alticraniatus and $M$. phanluongi sp. nov. $M$. s. thaianus was described from "Chengmai: city on the Meping, North Siam" (Shamel, 1942), presumably corresponding to Chiang Mai, N Thailand. This type locality is in relatively close proximity to that of $M$. phanluongi $\mathbf{s p .}$ nov. However, according to the original description (Shamel, 1942), M. s. thaianus is smaller (forearm 31.6 $\mathrm{mm}, \mathrm{CBL} 11.0 \mathrm{~mm}, \mathrm{CM} 34.6 \mathrm{~mm}$ ), but has a wider braincase and interorbital constriction (6.2 and $3.2 \mathrm{~mm}$, respectively) and contrastingly narrower rostrum (M3M3 $4.2 \mathrm{~mm}$ ). These measurements are in greater agreement with those provided by Bates \& Harrison (1997) for specimens of $M$. siligorensis from India and Nepal: CCL 11.2, CM3 4.5-4.8, M3M3 4.8-5.0. Hence the data currently available suggest the distinction of $M$. phanluongisp. nov. from both of the above subspecies.

M. phanluongi sp. nov. is readily distinguished from the remainder Myotis species occurring in the Indochinese Division (sensu Corbet \& Hill, 1992) in having significantly smaller external and cranial size, noticeably weaker dentition, and distinctive cusp pattern of lower molars and bacular shape.

Morphometric comparisons. A two-dimensional projection of the first and third principal components clearly segregates $M$. panluongi from all comparative material (Fig. 5). PC1 is highly correlated with rostral width, toothrow length and dentition massiveness, 


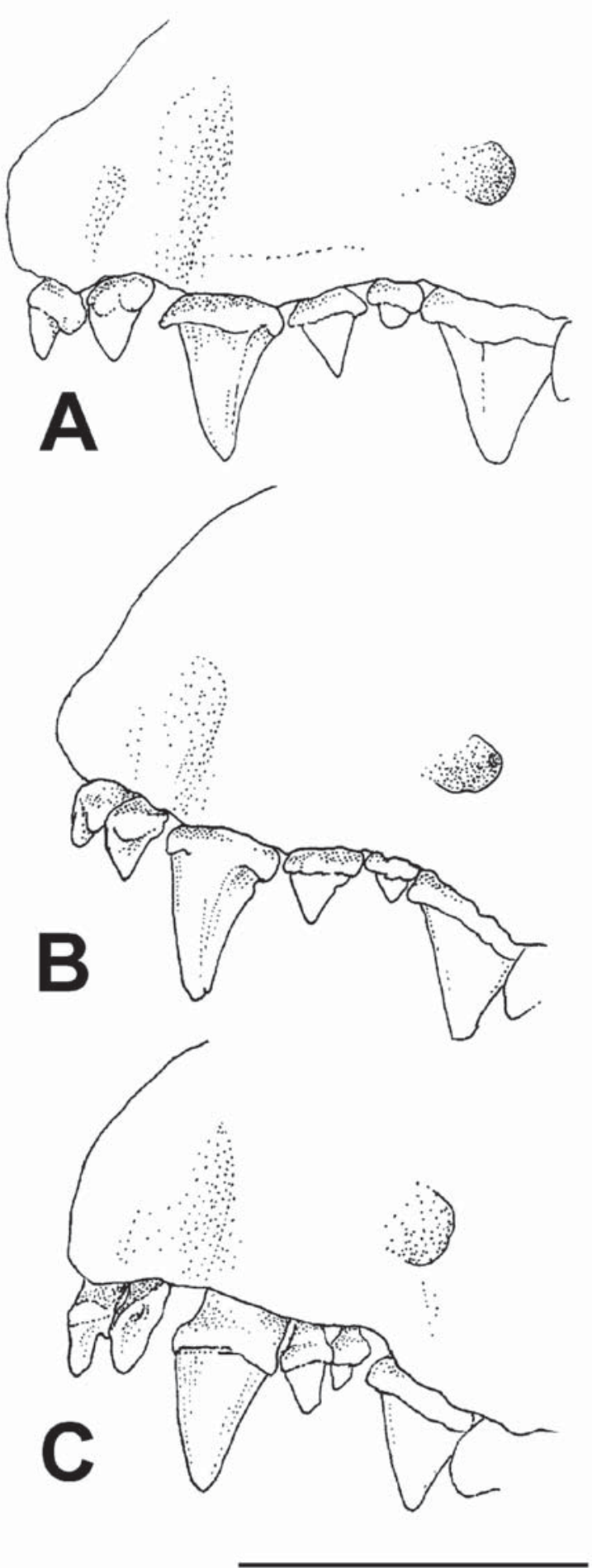

Figure 4. Camera lucida tracings of the anterior dentition of small Vietnamese Myotis (lateral view, facing towards left). A - Myotis phanluongi sp. nov., f, S-175154; B - Myotis annamiticus, m, S-167126; C - Myotis siligorensis, m, S167188. Scale bar is $1 \mathrm{~mm}$.
Table 2. Results of principal component analyses of 22 cranial measurements of 48 specimens of Myotis. See text for measurement abbreviations.

\begin{tabular}{|c|c|c|c|}
\hline Factor & 1 & 2 & 3 \\
\hline Eigenvalue & 16.604 & 1.884 & 1.058 \\
\hline$\%$ of total variance & 75.47 & 8.57 & 4.81 \\
\hline \multicolumn{4}{|c|}{ Component loadings: } \\
\hline CBL & 0.657 & 0.495 & 0.493 \\
\hline CCL & 0.641 & 0.483 & 0.534 \\
\hline RostrL & 0.479 & 0.646 & 0.117 \\
\hline $\mathrm{OccH}$ & 0.421 & 0.349 & 0.783 \\
\hline TympW & 0.355 & 0.322 & 0.767 \\
\hline BrainW & 0.420 & 0.369 & 0.785 \\
\hline IOrbW & 0.324 & 0.166 & 0.891 \\
\hline RostrW & 0.764 & 0.315 & 0.438 \\
\hline Pseud & -0.189 & 0.905 & 0.249 \\
\hline C_M3 & 0.694 & 0.522 & 0.417 \\
\hline P4_M3 & 0.829 & 0.222 & 0.412 \\
\hline C_crown & 0.752 & 0.217 & 0.422 \\
\hline C_P4 & 0.496 & 0.799 & 0.221 \\
\hline C_C & 0.660 & 0.261 & 0.551 \\
\hline M3_M3 & 0.390 & 0.138 & 0.237 \\
\hline M2 & 0.882 & -0.014 & 0.194 \\
\hline Mnd-gl & 0.675 & 0.425 & 0.495 \\
\hline Mnd-ang & 0.674 & 0.415 & 0.507 \\
\hline c_m3 & 0.732 & 0.496 & 0.393 \\
\hline p4_m3 & 0.829 & 0.126 & 0.355 \\
\hline c_p4 & 0.297 & 0.826 & 0.283 \\
\hline Coron & 0.559 & 0.162 & 0.480 \\
\hline
\end{tabular}

whereas PC3 is most influenced by braincase width (Tab. 2), suggesting that corresponding measurements are of particular identification value. Contrarily, pseudodiastem length (most strongly correlated with PC2) is not useful in separating $M$. phanluongi sp. nov. from M. s. alticraniatus, M. annamiticus, and M. laniger (graph not shown). Discriminant Functions were able to separate all five species from each other. Both posterior probabilities and squared Mahalanobis distances indicate relative similarity between $M$. s. alticraniatus and $M$. phanluongi sp. nov., while the latter 


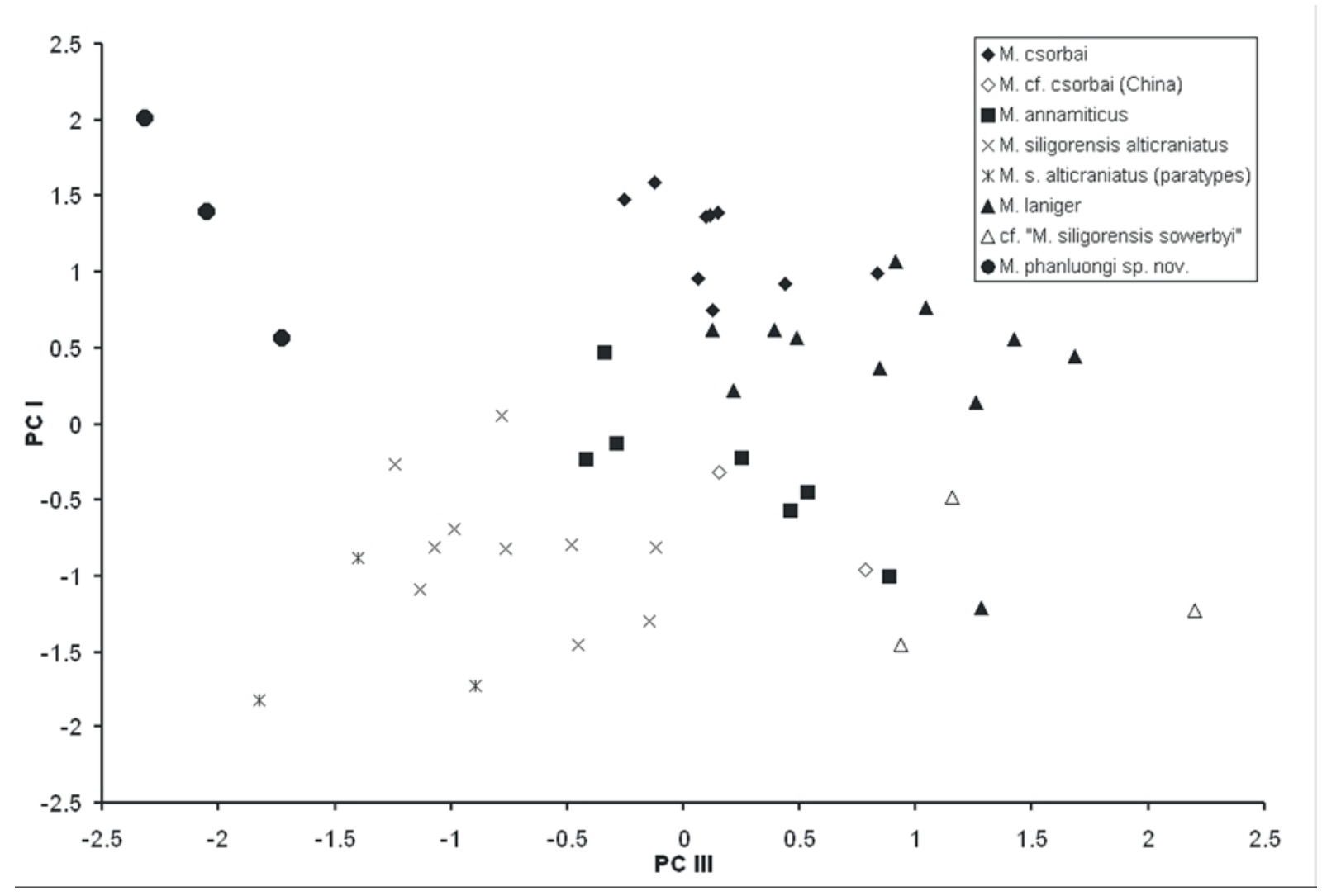

Figure 5. Bivariate scatterplot of the first and third Principal Components, calculated on the basis of 22 cranial measurements for 48 specimens of Myotis. See Tab. 2 for eigenvalues and component loadings.

remains distant from all a priory defined groups. Unfortunately, the small sample size available for M. phanluongi sp. nov. renders DF calculations unreliable; hence no data is presented here.

Molecular comparisons. Neighbour-joining analysis of 12S rDNA sequences (Fig. 6) corroborates the observed morphological similarity between $M$. phanluongi sp. nov. and M. siligorensis. M. phanluongi sp. nov. was more genetically divergent from sympatrically occurring $M$. siligorensis $(2.0 \%)$, than two specimens of M. siligorensis from localities situated over $1100 \mathrm{~km}$ apart (1.6\%). The next nearest neighbours to $M$. phanluongi sp. nov. are $M$. annamiticus and $M$. csorbai (4.0\% and $5.7 \%$ divergence, respectively). Despite the fact that genetic distances between $M$. phanluongi $\mathbf{s p . ~ n o v . ~ a n d ~} M$. siligorensis are significantly less than the average between species within Myotis, they may indicate the lack of recent introgression between sympatrically occurring populations of the two species. Although not unambiguously supportive of the species status of $M$. phanluongi sp. nov., $12 \mathrm{~S}$ data do not contradict the observed morphological and ecological (see below) differences and are also in agreement with DNA barcodes, which clearly separate the new species from all Myotis species analyzed to date using the mitochondrial cytochrome oxidase subunit I gene (Francis, Eger, Guillen, Kruskop, Borisenko, in litt.).
Results of the maximum parsimony analysis (Fig. 7) suggest that $M$. phanluongi sp. nov., $M$. annamiticus, $M$. siligorensis, and $M$. csorbai form a monophyletic clade, with $M$. capaccinii as a sister group. This is contradictory to the traditional placement of the "siligorensis" species group with the subgenus Selysius (Tate, 1941; Ellerman \& Morrison-Scott, 1966; Koopman, 1994) and is in agreement with the more recent views of several authors (Ruedi \& Mayer, 2001; Hoofer \& Bussche, 2003; Bickham et al., 2004; Stadelmann et al., 2004; Simmons, 2005) who abandoned the designation of subgenera within Myotis altogether.

Taxonomic remarks. Based on molecular and qualitative morphological comparison $M$. phanluongi $\mathbf{s p .}$ nov. should be considered a member of the "Myotis siligorensis" species group. This group was originally proposed by Tate (1941) for the single species - a point of view later supported by several authors, e.g., Koopman (1994). Although this species group is not defined in the latest mammal checklist (Simmons, 2005), we find it to be an operational taxonomic designation, encompassing small generalized Southeast Asian Myotis with low rostral part of the skull, elevated braincase and short canines. Comparison with morphologically similar species shows that one of the most prominent distinguishing morphological features of this species group is the cusp pattern of lower molars, exclusively 


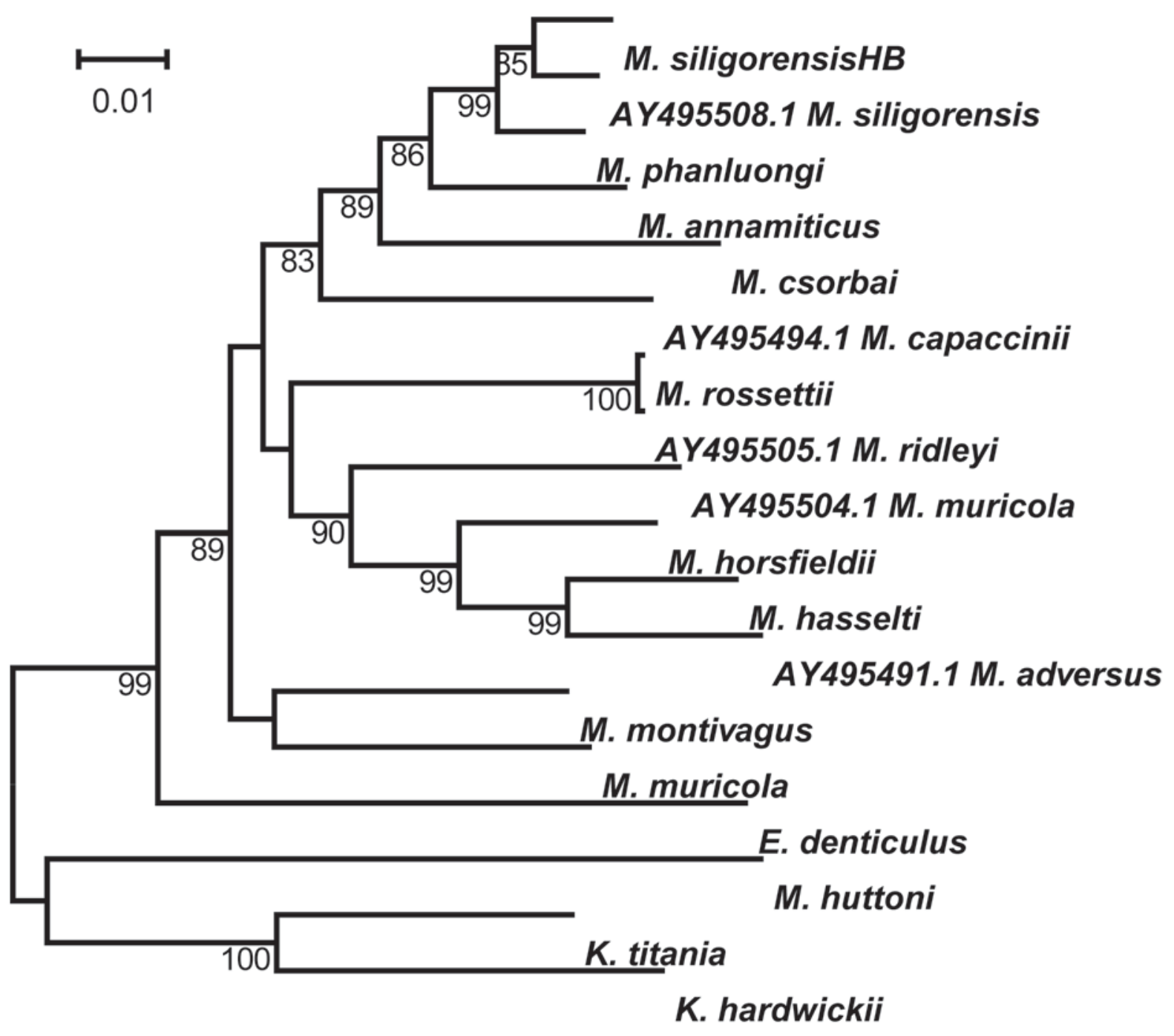

Figure 6. Neighbor-Joining tree constructed on the basis of $12 \mathrm{~S}$ rDNA sequences of 18 bat specimens. Bootstrap values below $70 \%$ are not shown. See text for explanations.

rare in Myotis which otherwise are "strict" myotodonts (Menu, 1987; Rosina, 2002). A similar dental structure with seminyctalodont M1 and seminyctalodont or submyotodont M2 can be found in extant genera Lasionycteris (Menu, 1987) and Murina (Rosina, 2003) and was used as substantiation for the description of a new fossil vespertilionid genus Submyotodon (Ziegler, 2003).

Otherwise both morphological and molecular data suggest close similarity of the "siligorensis" species group with $M$. annamiticus and $M$. csorbai (and possibly also with $M$. laniger) which demonstrate more prominent features of the "Leuconoe" morphotype (Koopman, 1994; Kruskop \& Tsytsulina, 2001). These characters include well-developed paraconules and pre-protocristae on M1-2, supplementary cusps on inner incisors (I2), slightly elongated cingulum of the upper canine, attachment of the wing membrane to the metatarsus proximal to its joint with the outer toe, relatively large foot, and narrow elongated ear pinna and tragus.
Ecological remarks. Bats from the type locality were collected and observed in proximity of small streams flowing through forested ravines at elevations of ca. $1250 \mathrm{~m}$ ASL. On April 20, 2003 they were observed foraging over the slope of a ravine with a small stream and above the stream itself. Bats foraging above the slope proceeded downstream and along the ravine within few meters away from vegetation and returned back within several minutes. Near the stream they followed along the current and remained close to the water, eventually stopping to circle around small pools formed by the streambed. Three individuals were scooped with a mobile trap; however, none were caught in mist net set up across the stream. Another specimen was captured on April 25, 2003 with a mobile trap over a small stream flowing along a ravine at ca. $1300 \mathrm{~m}$ ASL. Small dark bats flying just above the water surface were also observed over small streams near the top of Hon Ba Mountain (ca. 1500 m ASL), however, none 


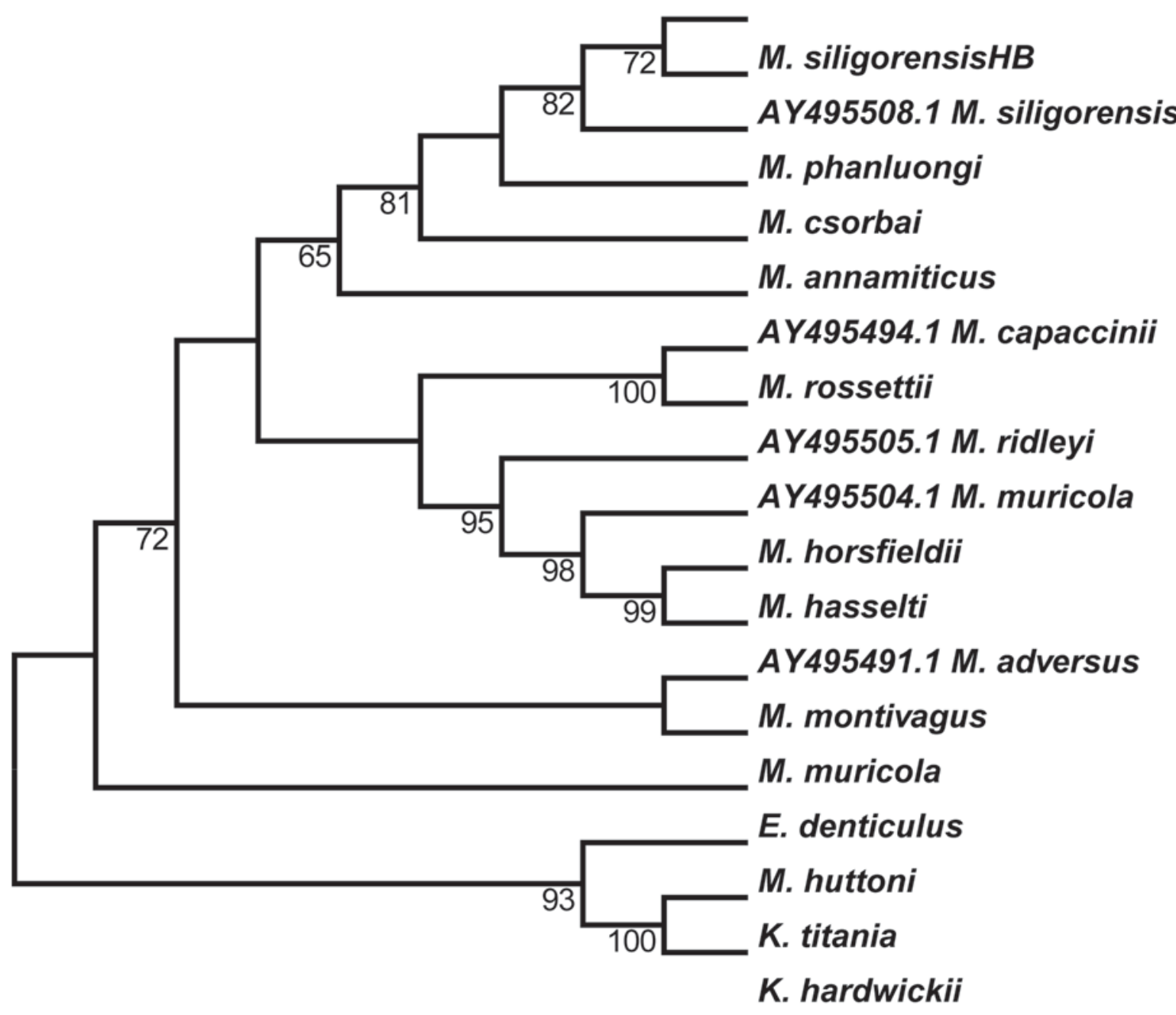

Figure 7. Maximum Parsimony tree constructed on the basis of 12S rDNA sequences of 18 bat specimens. Bootstrap values below $50 \%$ are not shown. See text for explanations.

were captured. While foraging, M. phanluongi $\mathbf{s p . ~ n o v . ~}$ emitted low-amplitude FM echolocation signals with maximum energy around $45 \mathrm{kHz}$ (estimated using a D100 heterodyning ultrasound detector, Pettersson Elektronik $\mathrm{AB}$ ). The lack of karst and known caves in the area suggests that, unlike the recently described $M$. annamiticus, $M$. phanluongi $\mathbf{s p . ~ n o v . ~ i s ~ n o t ~ a s s o c i a t e d ~}$ with such shelters. The females collected were each carrying a single near-term embryo; the male appeared reproductively inactive.

The coexistence of two morphologically and genetically discrete forms of small Myotis within the same geographic area deserves special attention not only from the taxonomic standpoint, but also as a possible model of ecological speciation. There seem to be no major physical barriers preventing the intermixing of bat populations from the foothills and those from the ridge of Hon $\mathrm{Ba}$, because both collection sites are located within the basin of the same river system and within ca. $7 \mathrm{~km}$ of straight flying distance. Altitudinal gradient alone is insufficient to explain their segrega- tion, because $M$. siligorensis has been reported to occur up to elevations of 2770 m ASL (Bates \& Harrison, 1997), hence we should like to draw attention to the differences between the habitats where the two species were observed.

The area around the type locality of $M$. phanluong $i$ sp. nov. is covered by moderately disturbed mature polydominant montane lowland forest (Kuznetsov et al., 2006), which underwent selective logging in some local parts, however, retained the bulk of its original forest stand (Fig. 8). The streams lie at the bottom of deep ravines. Stream segments with fast current usually have a width of one to two meters; eventually they alternate with small shallow pools. Stone outcrops are few; however, talus piles with huge boulders are accumulated in some parts of the ravines, eventually forming huge interstitial cavities. In these places the surface current of streams terminates and the water flows under the rocks, up to several meters below the surface. The regimen of these streams appears to be relatively stable compared to rivers at the foothills, but also displays 


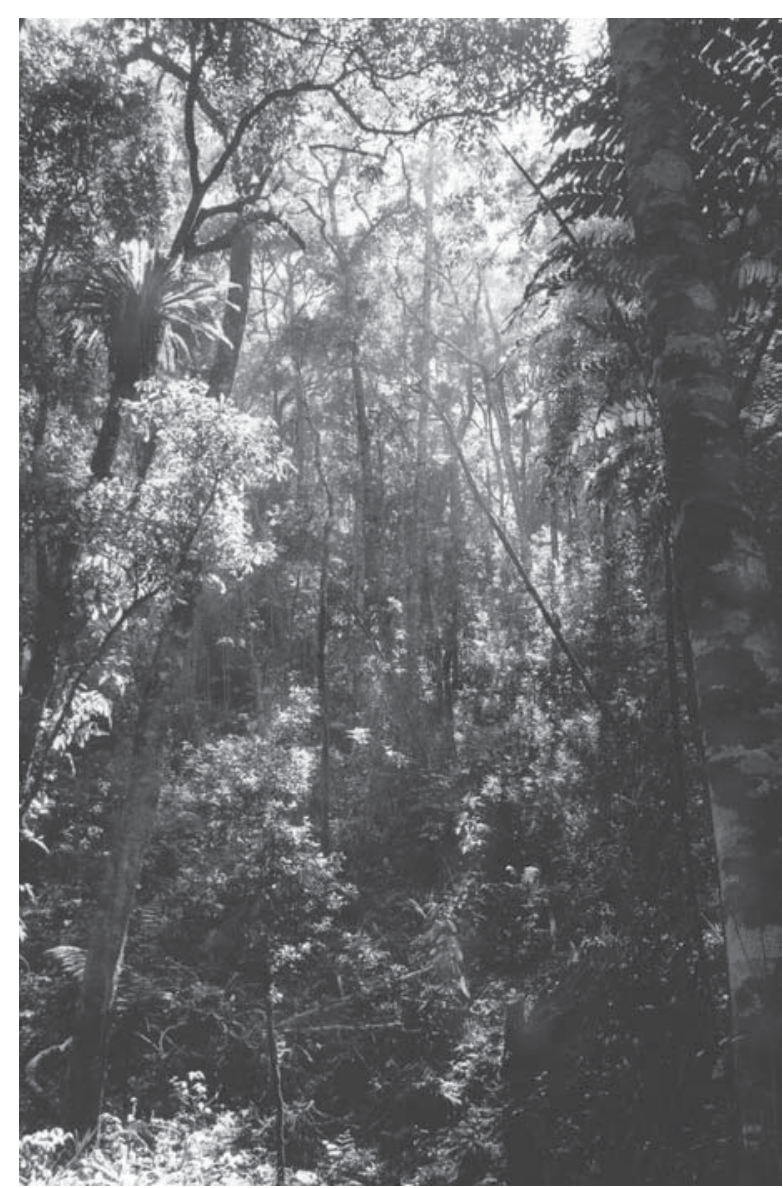

Figure 8. Mountain ravine at higher elevations of Hon Ba Mountain (ca. $1250 \mathrm{~m} \mathrm{ASL}$ ): type locality of Myotis phanluongi sp. nov.

certain variation in response to local rainfall. The specimen collected in the Da Nim River valley ca. $30 \mathrm{~km}$ away from the type locality was also taken near a stream at the edge of mature forest, although the terrain at this site was less montane.

$M$. siligorensis alticraniatus were collected in the foothills of Hon Ba in several locations along Suoi Dau River and its right tributary $\left(12.123^{\circ} \mathrm{N}, 109.002^{\circ} \mathrm{E}\right.$, $220 \mathrm{~m}$ ASL). The right bank side of Suoi Dau is a steep mountain slope covered by heavily disturbed bamboodominated formations with remains of polydominant semievergreen lowland forest (Kuznetsov et al., 2006), whereas the left bank side is less hilly, completely deforested and occupied by agricultural land (cashew plantations). The riverbed is ca. 10 to $30 \mathrm{~m}$ wide, rocky with rapids alternating with reaches, and numerous large boulders scattered throughout. The tributary within several $\mathrm{km}$ upstream from its fusion with Suoi Dau $\left(12.103^{\circ} \mathrm{N}, 109.000^{\circ} \mathrm{E}, 250 \mathrm{~m} \mathrm{ASL}\right)$ is a moderatesized mountain stream flowing along a small gorge through basalt outcrops. Due to significant altitudinal drop, it is formed by a series of waterfalls or rapids alternating with pools of relatively calm water up to tens of meters long, several meters wide and, occasion-

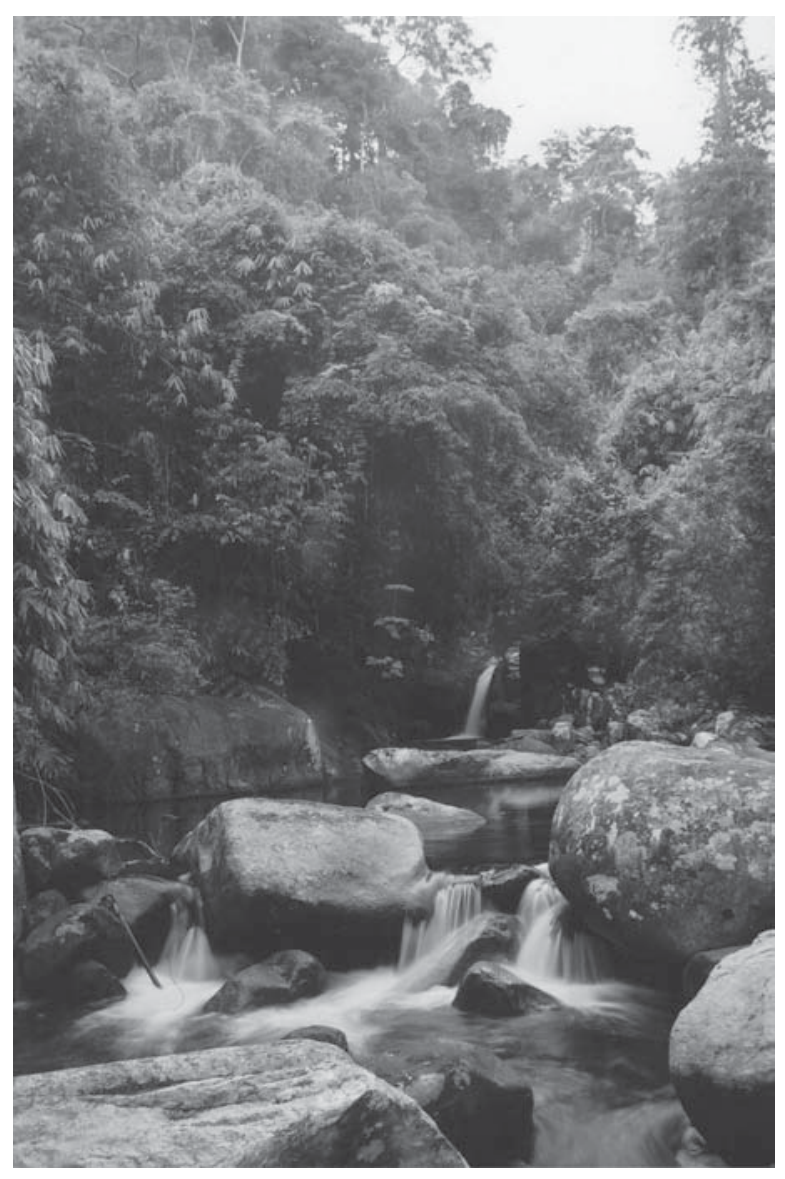

Figure 9. Stream at the foothills of Hon Ba Mountain (ca. 250 m ASL): typical habitat of Myotis siligorensis alticraniatus.

ally, several meters deep (Fig. 9). Plant formations in the immediate vicinity are overwhelmingly dominated by thin bamboo (ca. $5 \mathrm{~cm}$ or less in stem diameter), with individual trees visible higher up the slopes. The surrounding area is not cultivated; however, it apparently underwent extensive logging several decades ago. The regimen of both these rivers is supposedly unstable and heavily influenced by rainfall and catchment conditions upstream.

The observed foraging behaviour of both M. s. alticraniatus and $M$. phanluongi sp. nov. in Hon Ba resemble those of M. csorbai (Topal, 1997; Csorba et al., 1999) and M. annamiticus (Kruskop \& Tsytsulina, 2001). Interestingly, it contrasts with observations provided by Bates \& Harrison (1997: 127) for Nepal, who report that $M$. siligorensis "was seen flying high in the air but also frequently entered houses at night to hunt near lamps". This behaviour is in better agreement with observations of M. siligorensis in Minh Khoa, Quan Binh Province, Vietnam (SVK, in litt.), where they were captured with a flap trap in a village while flying ca. 4-8 $\mathrm{m}$ above the ground. The preference of $M$. $s$. alticraniatus for disturbed habitat is in general agreement with previous findings. Because in the Hon $\mathrm{Ba}$ 
foothills it lives in the immediate vicinity of cultivated land, it is possible that it may also use agricultural habitats, although no observations are yet available to confirm this. On the contrary, the collecting localities of $M$. phanluongi sp. nov. appear to be confined to areas presently or very recently occupied by mature montane forest. This instantly raises potential conservation concerns, in view of the intense deforestation and infrastructure development presently carried out in the Bi Doup-Hon Ba area. Understanding that available anecdotal observations are insufficient to draw ecological conclusions, we think that they may be useful in designing future ecological studies of the new species and determining its distribution range, status and conservation requirements.

ACKNOWLEDGEMENTS. Field studies in Hon Ba were carried out within the framework of the "Ecolan" program, with support from the Joint Vietnam-Russian Tropical Research and Technological Centre and organized by leaders of the research team Phan Luong and A.N. Kuznetsov. The administrative part of preparing the expedition was coordinated by the Coastal Branch of the Vietnam-Russian Tropical Centre (Nha Trang), Co-directors Nguyen Van Doan and V.K. Nezdoliy. Various help in the field was provided by S.P. Kuznetsova, M.V. Kalyakin, A.L. Monastyrskii, V.V. Bobrov, Nguyen Huu Thuc, Nguyen Van Thinh, Nguyen Dang Hoi, Nguyen Duc Anh, and Vu Van Lien. V.S. Lebedev and S.J. Adamowicz provided helpful comments during manuscript preparation.

Processing of the collection materials was done at the ZMMU, using the collection facilities of the Theriological Section with support from I.Ja. Pavlinov and O.L. Rossolimo and at the ROM with permission and support from J.L. Eger and B.K. Lim. The examination of FMNH specimens, particularly the type series of $M$. siligorensis alticraniatus was done at the ROM and made possible due to a loan to J.L. Eger kindly arranged by L.R. Heaney. Images of the type specimen of M. siligorensis from the British Museum (Natural History) were kindly provided by C.M. Francis, who made them with permission from P.D. Jenkins.

12 S DNA was analyzed at the Engelhardt Institute of Molecular Biology with support from A.B. Poltaraus. DNA barcodes were generated at the Biodiversity Institute of Ontario with support to P.D.N. Hebert from Genome Canada through the Ontario Genomics Institute, the Canada Foundation for Innovation, the Ontario Innovation Trust, the Canada Research Chairs Program and NSERC.

\section{References}

American Society of Mammalogists Animal Care and Use Committee. 1998. Guidelines for the capture, handling, and care of mammals as approved by the American Society of Mammalogists // Journal of Mammalogy, Vol.79. No.4. P.1416-1431.

Bates P.J.J. \& Harrison, D.L. 1997. Bats of the Indian Subcontinent. Sevenoaks, Kent: Harrison Zoological Museum. 258 p.

Bickham J.W. Patton J.C. Schlitter D.A. Rautenbach I.L. \& Honeycutt R.L. 2004. Molecular phylogenetics, karyotypic diversity, and partition of the genus Myotis (Chi- roptera: Vespertilionidae) // Molecular Phylogenetics and Evolution. Vol.33. No.2. P.333-338.

Borisenko A.V., Kruskop S.V. \& Morozov P.N. 2006. [Preliminary inventory of the mammal fauna of the southeastern extremity of the Truong Son mountain range] // Korzoun L.P., Rozhnov V.V. \& Kalyakin M.V. (eds.). [Materials of Zoological and Botanical Studies in Bi Doup and Hon Ba Mountain, Dalat Plateau, Southern Vietnam.] Moskva-Hanoi: KMK. P.159-194 [in Russian with English summary].

Borisenko A.V., Lim B.K., Ivanova N.V., Hanner R.H. \& Hebert P.D.N. 2008. DNA barcoding in surveys of small mammal communities: A field study In Suriname // Molecular Ecology Resources. Vol.8. No.3. P.471-479.

Borisenko A.V. 1999. [A mobile trap for capturing bats in flight] // Plecotus et al. Vol.2. P.10-19 [in Russian, with English summary].

Borisenko A.V. \& Kruskop S.V. 2003. Bats of Vietnam and Adjacent Territories. An Identification Manual. Moscow: Geos. 203 pp.

Clare E.L., Lim B.K., Engstrom M.D., Eger J.L. \& Hebert P.D.N. 2007. DNA barcoding of Neotropical bats: species identification and discovery within Guyana // Molecular Ecology Notes. Vol.7. P.184-190.

Corbet G.B. \& Hill J.E. 1992. The Mammals of the Indomalayan Region. Oxford: Oxford University Press. 488 p.

Csorba G., Kruskop S.V. \& Borissenko A.V. 1999. Recent records of bats (Chiroptera) from Nepal, with remarks on their natural history // Mammalia. Vol.63. No.1. P.61-78.

Ellerman J.R. \& Morrison-Scott T.C.S. 1966. Checklist of Palaearctic and Indian Mammals 1758 to 1946. Second Edition. London: British Museum (Natural History). 810 p.

Hajibabaei M., deWaard J.R., Ivanova N.V., Ratnasingham S., Dooh R.T., Kirk S.L., Mackie P.M. \& Hebert P.D.N. 2005. Critical factors for assembling a high volume of DNA barcodes // Philosophical Transactions of the Royal Society of London, Series B: Biological Sciences. Vol.360. P.1959-1967.

Hall T.A. 1999. BioEdit: a user-friendly biological sequence alignment editor and analysis program for Windows 95/ 98/NT // Nucleic Acids Symposium Series. Vol.41. P.95-98.

Hoofer S.R. \& Bussche R.A., van den. 2003. Molecular phylogenetics of the chiropteran family Vespertilionidae // Acta Chiropterologica. Vol.5 supplement. P.1-63.

Ivanova N.V., deWaard J.R. \& Hebert P.D.N. 2006. An inexpensive, automation-friendly protocol for recovering high-quality DNA // Molecular Ecology Notes. Vol.6. P.998-1002.

Ivanova N.V., Zemlak T.S., Hanner R.H. \& Hebert P.D.N. 2007. Universal primer cocktails for fish DNA barcoding // Molecular Ecology Notes. Vol.7. P.544-548.

Jones C., McShea W.J., Conroy M.J. \& Kunz T.H. 1996. Capturing Mammals // Wilson D.E. et al. (eds.) Measuring and Monitoring Biological Diversity. Standard Methods for Mammals. Washington and London: Smithsonian Institution Press. P.115-155.

Koopman K.F. 1994. Chiroptera: Systematics. Berlin: Walter and Gruyter Co. 217 p. 
Kruskop S.V. \& Tsytsulina K.A. 2001. A new big-footed mouse-eared bat (Myotis, Vespertilionidae, Chiroptera) from Vietnam // Mammalia. Vol.62. No.1. P.63-72.

Kuznetsov A.N., Kuznetsova S.P. \& Luong P. 2006. [The forest vegetation on the mountain massives Bi Dup and Hon $\mathrm{Ba}$, southern part of the main Hymalayan ridge Chuong Son] // Korzoun L.P., Rozhnov V.V. \& Kalyakin M.V. (eds.) [Materials of Zoological and Botanical Studies in Bi Doup and Hon Ba Mountains, Da Lat Plateau, Southern Vietnam.] Moskva-Hanoi: KMK. P.9115 [in Russian with English summary].

Kuznetsov G.V., Kulikov E.E., Petrov N.B., Ivanova N.V., Lomov A.A., Kholodova M.V. \& Poltaraus A.B. 2001. The "Linh Duong" Pseudonovibos spiralis (Mammalia, Artiodactyla) is a new buffalo // Naturwissenschaften. Vol.88. P.123-125.

Menu H. 1987. Morphotypes dentaires actuels et fossiles des chiroptères // Palaeovertebrata. Vol.17. P.77-150.

Pfunder M., Holzgang O. \& Frey J.E. 2004. Development of microarray-based diagnostics of voles and shrews for use in biodiversity monitoring studies, and evaluation of mitochondrial cytochrome oxidase I vs. cytochrome $b$ as genetic markers // Molecular Ecology. Vol.13. P.12771286.

Rosina V.V. 2002. [Odontology of the mouse-eared bats (Myotis) of the Palaearctic] // Plecotus et al. Vol.5. P.11-27 [in Russian with English summary].

Rosina V.V. 2003. [Murinodontia - the special type of lower molar cusps morphology in bats] // Plecotus et al. Vol.6. P.3-6 [in Russian with English summary].

Ruedi M. \& Mayer F. 2001. Molecular systematics of bats of the genus Myotis (Vespertilionidae) suggests determinis- tic ecomorphological convergences // Molecular Phylogenetics and Evolution. Vol.21. No.3. P.436-448.

Shamel H.H. 1942. A collection of bats from Thailand (Siam) // Journal of Mammalogy. Vol.23. P.317-328.

Simmons N.B. 2005. Order Chiroptera // Wilson D.E. \& Reeder D.M. (eds.) Mammal Species of the World: A Taxonomic and Geographic Reference, Third Edition. Baltimore: Johns Hopkins University Press. P.312-529.

Springer M., Hollar L. \& Burk A. 1995. Compensatory substitutions and the evolution of the mitochondrial $12 \mathrm{~S}$ rRNA gene in mammals // Molecular Biology and Evolution. Vol.12. P.1138-1150.

Stadelmann B., Jacobs D.S., Schoeman C. \& Ruedi M. 2004. Phylogeny of African Myotis bats (Chiroptera, Vespertilionidae) inferred from cytochrome b sequences // Acta Chiropterologica. Vol.6. No.2. P.177-192.

Tamura K., Dudley J., Nei M. \& Kumar S. 2007. MEGA4: Molecular Evolutionary Genetics Analysis (MEGA) Software Version 4.0 // Molecular Biology and Evolution. Vol.24. P.1596-1599.

Tate G.H.H. 1941. A review of the genus Myotis (Chiroptera) of Eurasia: with special reference to species occurring in the East Indies // Bulletin of the American Museum of Natural History. Vol.78. No.8. P.537-565.

Topal G. 1997. A new mouse-eared bat species, from Nepal, with statistical analyses of some other species of subgenus Leuconoe (Chiroptera: Vespertilionidae) // Acta Zoologica Academiae Scientiarum Hungaricae. Vol.43. No.4. P.375-402.

Ziegler R. 2003. Bats (Chiroptera, Mammalia) from Middle Miocene karstic fissure fillings of Petersbuch near Eichstätt, Southern Franconian Alb (Bavaria) // Geobios. Vol.36. No.4. P.447-490. 